\title{
Panorama das manifestações bucais decorrente do tratamento do câncer infantil:
}

\section{Uma revisão integrativa}

\author{
Overview of oral manifestations resulting from the treatment of childhood cancer: An integrative \\ review
}

Resumen de las manifestaciones orales resultantes del tratamiento del cáncer infantil: Una revisión integradora

Recebido: 11/06/2021 | Revisado: 22/06/2021 | Aceito: 01/07/2021 | Publicado: 14/07/2021

Larissa Silva Costa

ORCID: https://orcid.org/0000-0003-3802-2603 Universidade Metropolitana de Santos, Brasil E-mail: lary017silva@gmail.com

Maria Salete Nahás Pires Côrrea ORCID: https://orcid.org/0000-0002-3607-7152 Universidade de São Paulo, Brasil

E-mail: msnpcorr@usp.br

José Carlos P. Imparato

ORCID: https://orcid.org/0000-0002-1990-2851 Universidade de São Paulo, Brasil

E-mail: jimparato@usp.br

Karla Mayra Rezende

ORCID: https://orcid.org/0000-0003-4340-0699 Universidade de São Paulo, Brasil

Universidade Metropolitana de Santos, Brasil

E-mail: karla.rezende@usp.br

\begin{abstract}
Resumo
Crianças submetidas ao tratamento oncológico apresentam muitos sintomas e doenças que se manifestam na de maneira aguda e crônica. O objetivo do presente trabalho foi realizar uma revisão integrativa da literatura sobre a seguinte pergunta clinica: Os tratamentos oncológicos (quimioterapia e radioterapia) em crianças podem ter repercussões bucais? Realizou-se uma pesquisa de artigos científicos na base de dados Medline, sem a utilização de limites temporais. Foram considerados as publicações disponibilizadas na íntegra e em inglês. Dos 399 títulos identificados, 37 artigos atenderam aos critérios de inclusão e foram incluídos na revisão. Foram removidos 11 artigos que não atendiam o desfecho ou estava de acesso fechado e sem retorno dos autores, finalizando 26 artigos para estudo final. Há evidências significativas de que crianças e adolescentes apresentam numerosos e complexos sintomas e problemas durante e após o tratamento do câncer. As doenças podem ser manifestadas durante o tratamento como mucosite, ulcerações, xerostomia, cárie e doença periodontal. Para essas doenças o uso de laser, orientação do dentifrício fluoretado e restaurações devem ser realizados para melhorar na alimentação e recuperação da saúde e, ao longo prazo, de acordo com a dose, tempo e medicação, o tratamento promove atraso no crescimento da criança, e consequentemente o tempo de erupção dentária e anomalias dentárias. Portanto, a compreensão da trajetória dos sintomas, alicerçada nas vivências da criança e da família, é fundamental para o cuidado integral e sensível à criança com câncer e sua família, sendo o odontopediatra profissional fundamental para acompanhar e tratar essas crianças até sua fase adulta.
\end{abstract}

Palavras-chave: Oncologia; Odontopediatria; Manifestações orais.

\begin{abstract}
Children undergoing cancer treatment have many symptoms and diseases that manifest themselves in an acute and chronic manner. The aim of this study was to carry out an integrative literature review on the following clinical question: Can cancer treatments (chemotherapy and radiotherapy) in children have oral repercussions? A search of scientific articles in the Medline database was carried out, without the use of time limits. Publications made available in full and in English were considered. Of the 399 titles identified, 37 articles met the inclusion criteria and were included in the review. Eleven articles that did not meet the outcome or had closed access and no return from the authors were removed, finalizing 26 articles for the final study. There is significant evidence that children and adolescents experience numerous and complex symptoms and problems during and after cancer treatment. Diseases can be manifested during treatment as mucositis, ulcerations, xerostomia, caries and periodontal disease. For these diseases, the use of laser, orientation of fluoridated toothpaste and restorations should be performed to improve
\end{abstract}


nutrition and health recovery and, in the long term, according to the dose, time and medication, the treatment promotes delay in the child's growth, and consequently the time of tooth eruption and tooth anomalies. Therefore, understanding the trajectory of symptoms, based on the experiences of the child and family, is essential for comprehensive and sensitive care for children with cancer and their families, with the professional pediatric dentist being essential to monitor and treat these children until their adulthood.

Keywords: Oncology; Pediatric dentistry; Oral manifestations.

\begin{abstract}
Resumen
Los niños que se someten a un tratamiento contra el cáncer tienen muchos síntomas y enfermedades que se manifiestan de forma aguda y crónica. El objetivo de este estudio fue realizar una revisión integradora de la literatura sobre la siguiente pregunta clínica: ¿Pueden los tratamientos oncológicos (quimioterapia y radioterapia) en niños tener repercusión oral? Se realizó una búsqueda de artículos científicos en la base de datos Medline, sin uso de límites de tiempo. Se consideraron las publicaciones disponibles en su totalidad y en inglés. De los 399 títulos identificados, 37 artículos cumplieron los criterios de inclusión y se incluyeron en la revisión. Se eliminaron once artículos que no cumplieron con el resultado o que tenían acceso cerrado y sin retorno de los autores, finalizando 26 artículos para el estudio final. Existe evidencia significativa de que los niños y adolescentes experimentan numerosos y complejos síntomas y problemas durante y después del tratamiento del cáncer. Las enfermedades pueden manifestarse durante el tratamiento como mucositis, ulceraciones, xerostomía, caries y enfermedad periodontal. Para estas enfermedades se debe realizar el uso de láser, orientación de dentífrico fluorado y restauraciones para mejorar la nutrición y la recuperación de la salud y, a largo plazo, según la dosis, el tiempo y la medicación, el tratamiento promueve el retraso en el crecimiento del niño, y, en consecuencia, el momento de la erupción y las anomalías dentarias. Por tanto, comprender la trayectoria de los síntomas, a partir de las vivencias del niño y la familia, es fundamental para la atención integral y sensible del niño con cáncer y sus familias, siendo fundamental el odontopediatra profesional para el seguimiento y tratamiento de estos niños hasta la edad adulta.
\end{abstract}

Palabras clave: Oncología; Odontología pediátrica; Manifestaciones orales.

\title{
1. Introdução
}

A Odontopediatria baseada em evidências envolve três pilares: aplicação apropriada da melhor ciência disponível, acumulo de conhecimento por meio de experiências clinicas e valores do paciente. O objetivo principal é sempre oferecer cuidados que otimizem resultados (Chi, 2017). No Brasil, segundo o Instituto Nacional de Câncer (INCA) são registrados 12 mil novos casos de câncer infantil ao ano. Os tipos mais comuns são as leucemias, tumores do sistema nervoso central, linfomas e tumores sólidos como o neuroblastoma, sarcomas e o tumor de Wilms.

O câncer infantil possui características próprias e bem diferentes em relação ao câncer em adultos. As células que sofrem a mutação no material genético não conseguem amadurecer como deveriam e permanecem com as características semelhantes da célula embrionária, multiplicando-se de forma rápida e desordenada. Por isso, a proliferação do tumor é mais rápida em crianças. Por outro lado, respondem melhor à quimioterapia aumentando $80 \%$ de cura.

A quimioterapia e/ou radioterapia para o tratamento do câncer pode causar muitos efeitos colaterais agudos e de longo prazo na cavidade bucal. Além disso, devido à imunossupressão que ocorre nos pacientes, qualquer fonte existente ou potencial de infecções orais/dentárias e/ou trauma de tecidos moles pode comprometer o tratamento médico, levando à morbidade, mortalidade e custos mais altos de hospitalização.

De ordem geral, as crianças sob esses tratamentos podem apresentar complicações de ordem gerais como insuficiência cardíaca, neurotoxicidade, nefrotoxicidade, comprometimento no crescimento, distúrbios hormonais e de ordens orais e dentárias devido o tratamento comprometendo a qualidade de vida (Effinger et al., 2014; Zubowska, Wyka, Fendler, Mlynarski, \& Zalewska-Szewczyk, 2013). De ordem bucal, uma grande parte das crianças submetidas ao tratamento oncológico, desenvolvem complicações devido à falta de prevenção, deficiência da higiene oral, mudança na alimentação e a destruição de células benignas da mucosa bucal.

Durante o tratamento pode manifestar algumas alterações na cavidade bucal, como: xerostomia, candidíase, aftas, sangramento gengival e mucosite, onde se o odontopediatra estiver inserido na equipe multidisciplinar, cuidará evitando 
comprometimentos do sistema imunológico (Ritwik \& Chrisentery-Singleton, 2020; Carvalho, Medeiros-Filho, \& Ferreira, 2018, LOPES, 2012). Assim, o objetivo desse trabalho foi fazer uma revisão integrativa das repercussões bucais que podem acontecer com crianças submetidas ao tratamento oncológico

\section{Metodologia}

O estudo trata-se de uma pesquisa qualitativa, realizado por meio de uma revisão integrativa de literatura (Estrela, 2018; Ludke, 2013).

A pesquisa e busca dos artigos foi realizada na base de dados Medline (PubMed). Os descritores foram escolhidos por sua importância na delimitação do tema de acordo com termos do Medical Subject Headings (MeSH). A última atualização aconteceu no dia 07 de junho de 2021. As seguintes palavras-chave foram empregadas: " (Child, Preschool OR children Or childhood) AND (chemotherapy OR radiotherapy) AND (dental abnormalities) OR (dental development) OR (pediatric dentistry)) OR (caries)) OR (mucositis)) OR (xerostomia) OR (aphthous stomatitis) OR (Candidiasis, Oral) AND (observational studies) OR (case report). Os dados foram selecionados de acordo com os seguintes critérios: trabalhos completos em língua inglesa que estivessem de acordo com a narrativa do tema e disponível de forma íntegra sobre câncer infantil e manifestações bucais. Para a escolha foram considerados os seguintes tipos de artigos: casos clínicos e estudos observacionais em crianças, submetidas ao tratamento oncológico. A seleção dos estudos foi realizada de maneira independente por dois revisores, por meio da plataforma de seleção Rayyan. Inicialmente os artigos foram selecionados após a leitura do título, resumo e os que atenderam os critérios de elegibilidade para então a leitura íntegra para inclusão ou exclusão na revisão. As discordâncias na fase da leitura na íntegra foram resolvidas por consenso por um terceiro revisor.

\section{Resultados e Discussão}

Foram encontrados 399 artigos, dos quais após a exclusão, 37 foram lidos. Os artigos analisados variaram sua publicação entre os anos de 1968 até 2021.

Ao fazer a leitura, foram excluídos 7 artigos porque não estava relacionado ao desfecho ou apenas descrevendo um caso clinico de criança submetida ao tratamento radioterápico ou quimioterápico não relacionando com as manifestações bucais. Outro ponto que fez a exclusão de quatro artigos, foram só estavam disponíveis o titulo ou resumo por serem artigos muito antigo. Mesmo assim, tentou-se entrar em contato com os autores responsáveis para pedir o texto, mas não se obteve retorno.

A Figura 1 ilustra o diagrama dos estudos selecionados.

Figura 1. Estudos selecionados.

\begin{tabular}{|l|l|}
\hline - Total de estudos encontrados na base de \\
dado Medline
\end{tabular}

Fonte: Autores. 
Research, Society and Development, v. 10, n. 8, e35510817072, 2021

(CC BY 4.0) | ISSN 2525-3409 | DOI: http://dx.doi.org/10.33448/rsd-v10i8.17072

Tabela 1. Descrição das características dos estudos incluídos neste trabalho.

\begin{tabular}{|c|c|c|c|c|c|c|c|c|c|}
\hline Número & Autores & Ano & $\begin{array}{l}\text { País } \\
\text { publicado }\end{array}$ & Título & $\mathbf{N}$ & $\begin{array}{l}\text { Idade das } \\
\text { crianças }\end{array}$ & $\begin{array}{l}\text { Etnia da } \\
\text { população } \\
\text { estudada }\end{array}$ & Tipo de câncer & Alterações bucais \\
\hline 1 & Juárez-Lópes et al. & 2018 & $\begin{array}{l}\text { Cidade do } \\
\text { México }\end{array}$ & $\begin{array}{l}\text { Oral diseases in children with } \\
\text { acute lymphoblastic leukemia } \\
\text { with chemotherapy treatment }\end{array}$ & 103 & 3-15 anos & $\begin{array}{l}\text { Não } \\
\text { mencionado }\end{array}$ & $\begin{array}{c}\text { Leucemia } \\
\text { linfobástica aguda }\end{array}$ & $\begin{array}{l}\text { Mucosite, candidíase, } \\
\text { gengivite, ulceras }\end{array}$ \\
\hline 2 & Kowlessar et al. & 2019 & $\begin{array}{l}\text { República } \\
\text { de } \\
\text { Trindade } \\
\text { e Tobago }\end{array}$ & $\begin{array}{l}\text { Oral health among children } \\
\text { attending an oncology clinic in } \\
\text { Trinidad }\end{array}$ & 71 & $1-15$ anos & $\begin{array}{c}\text { Não } \\
\text { mencionado }\end{array}$ & $\begin{array}{l}\text { Leucemia } \\
\text { linfobástica aguda } \\
+ \text { prevalente }\end{array}$ & carie, gengivite, mucosite \\
\hline 3 & Lucena et al. & 2019 & Brasil & $\begin{array}{l}\text { The contribution of motor } \\
\text { changes to oral mucositis in } \\
\text { pediatric cancer patients: a } \\
\text { cross-sectional study }\end{array}$ & 40 & 02 ate 19 & $\begin{array}{c}\text { Não } \\
\text { mencionado }\end{array}$ & $\begin{array}{l}\text { tumores } \\
\text { hematológicos }\end{array}$ & $\begin{array}{l}\text { Mucosite, candidíase, } \\
\text { gengivite, ulceras }\end{array}$ \\
\hline 4 & Guimaraes et al. & & Brasil & $\begin{array}{l}\text { The incidence of several oral } \\
\text { mucositits and its occurrence } \\
\text { sites in pediatric oncologic } \\
\text { patients }\end{array}$ & 56 & 01 ate 18 & $\begin{array}{c}\text { Não } \\
\text { mencionado }\end{array}$ & $\begin{array}{l}\text { Tumores } \\
\text { hematológicos e } \\
\text { sólidos }\end{array}$ & mucositie \\
\hline 5 & Peretz & 2014 & Israel & $\begin{array}{l}\text { Chemotherapy Induced Dental } \\
\text { Changes in a Child with } \\
\text { Medulloblastoma: A Case } \\
\text { Report }\end{array}$ & 1 & $\begin{array}{c}3 \text { anos e } 8 \\
\text { meses }\end{array}$ & branca & meduloblastoma & $\begin{array}{l}\text { raizes dentárias finas e cirtas, } \\
\text { microdontia, anodontia, lesao } \\
\text { de cárie, gengivite }\end{array}$ \\
\hline 6 & Farsi & 2016 & $\begin{array}{l}\text { Arabia } \\
\text { Saudita }\end{array}$ & $\begin{array}{l}\text { Children undergoing } \\
\text { chemotherapy: is it too late for } \\
\text { dental rehabilitation? }\end{array}$ & 1 & 5 & $\begin{array}{c}\text { Não } \\
\text { mencionado }\end{array}$ & neuroblastoma & $\begin{array}{l}\text { mucosite, lesao de carie, } \\
\text { candidose }\end{array}$ \\
\hline 7 & Rimulo & 2011 & Brasil & $\begin{array}{l}\text { Chemotherapy-induced oral } \\
\text { mucositis in a patient with } \\
\text { acute lymphoblastic leukaemia }\end{array}$ & 1 & 5 & Branca & leucemia & mucosite \\
\hline 8 & Fleming & 1991 & Dublin & $\begin{array}{l}\text { Dental management of the } \\
\text { pediatric oncology patient. }\end{array}$ & 1 & 3 & $\begin{array}{c}\text { Não } \\
\text { mencionado }\end{array}$ & leucemia & $\begin{array}{l}\text { desenvolvimento anormal } \\
\text { dental e craniofacial }\end{array}$ \\
\hline 9 & Zarina & 2005 & Malásia & $\begin{array}{l}\text { Dental abnormalities of a long- } \\
\text { term survivor of a childhood } \\
\text { hematological } \\
\text { malignancy: literature review } \\
\text { and report of a case }\end{array}$ & 1 & 10 & $\begin{array}{c}\text { Não } \\
\text { mencionado }\end{array}$ & $\begin{array}{c}\text { tumor } \\
\text { hematológico }\end{array}$ & $\begin{array}{l}\text { raizes finas, cárie, atraso na } \\
\text { erupçao e exfoliaçao, }\end{array}$ \\
\hline 10 & Venkataraghavan & 2013 & India & $\begin{array}{l}\text { Abnormal odontogenesis } \\
\text { following management of }\end{array}$ & 1 & 8 & $\begin{array}{c}\text { Não } \\
\text { mencionado }\end{array}$ & retinoblastoma & microdontia \\
\hline
\end{tabular}


Research, Society and Development, v. 10, n. 8, e35510817072, 2021

(CC BY 4.0) | ISSN 2525-3409 | DOI: http://dx.doi.org/10.33448/rsd-v10i8.17072

\begin{tabular}{|c|c|c|c|c|c|c|c|c|c|}
\hline & & & & $\begin{array}{l}\text { childhood cancer } \\
\text { (retinoblastoma): review and a } \\
\text { new variant }\end{array}$ & & & & & \\
\hline 11 & Jalali & 2012 & $\begin{array}{l}\text { Estados } \\
\text { Unidos }\end{array}$ & $\begin{array}{l}\text { Management of dental } \\
\text { complications in a child with } \\
\text { rhabdomyosarcoma }\end{array}$ & 1 & 5 & $\begin{array}{c}\text { Não } \\
\text { mencionado }\end{array}$ & rabdomiossarcoma & cárie, xerostomia \\
\hline 12 & Cheng & 2000 & Taiwan & $\begin{array}{l}\text { Effects of cancer therapy on } \\
\text { dental and maxillofacial } \\
\text { development in children: report } \\
\text { of case }\end{array}$ & 1 & 4 & $\begin{array}{c}\text { Não } \\
\text { mencionado }\end{array}$ & linfoma de burkitt & $\begin{array}{l}\text { atraso no crescimento } \\
\text { somático geral e ossos } \\
\text { maxilares, dentes com raízes } \\
\text { curtas e mal desenvolvidas }\end{array}$ \\
\hline 13 & Ariffin & 1999 & Malásia & $\begin{array}{l}\text { Successful treatment of } \\
\text { Candida albicans endocarditis } \\
\text { in a child with leukemia-case } \\
\text { report }\end{array}$ & 1 & 5 & $\begin{array}{c}\text { Não } \\
\text { mencionado }\end{array}$ & $\begin{array}{c}\text { Leucemia } \\
\text { linfobástica aguda }\end{array}$ & candidose \\
\hline 14 & Aielli & 2014 & Itália & $\begin{array}{l}\text { Rapid Resolution of Refractory } \\
\text { Chemotherapy-Induced Oral } \\
\text { Mucositis With Platelet Gel- } \\
\text { Released Supernatant in a } \\
\text { Pediatric Cancer Patient: A } \\
\text { Case Report }\end{array}$ & 1 & 5 & $\begin{array}{c}\text { Não } \\
\text { mencionado }\end{array}$ & rabdomiossarcoma & mucosite \\
\hline 15 & Hwang & 2011 & $\begin{array}{l}\text { Estados } \\
\text { Unidos }\end{array}$ & $\begin{array}{l}\text { Developmental Dental Defects } \\
\text { Linked with } \\
\text { Chemoradiotherapy: A Case } \\
\text { Report. }\end{array}$ & 1 & 8 & $\begin{array}{c}\text { Não } \\
\text { mencionado }\end{array}$ & rabdomiossarcoma & $\begin{array}{l}\text { agenesia dentária, alteração } \\
\text { radicular, microdontia, } \\
\text { mandibula subdesenvolvida, }\end{array}$ \\
\hline 16 & Yamada & 2009 & Japão & $\begin{array}{l}\text { Osteomyelitis of mandible } \\
\text { secondary to infantile } \\
\text { osteopetrosis: a case report }\end{array}$ & 1 & $\begin{array}{c}2 \text { anos e } 8 \\
\text { meses }\end{array}$ & $\begin{array}{c}\text { Não } \\
\text { mencionado }\end{array}$ & osteopetrose & osteomielite, abcesso dental \\
\hline 17 & Damm & 1988 & $\begin{array}{l}\text { Estados } \\
\text { Unidos }\end{array}$ & $\begin{array}{l}\text { Dentinal candidiasis in cancer } \\
\text { patients }\end{array}$ & 1 & $\begin{array}{c}2 \text { anos e } 3 \\
\text { meses }\end{array}$ & $\begin{array}{l}\text { Não } \\
\text { mencionado }\end{array}$ & leucemia & cárie, candidiase \\
\hline 18 & Svoboda & 1991 & $\begin{array}{l}\text { Estados } \\
\text { Unidos }\end{array}$ & $\begin{array}{l}\text { North American Burkitt's } \\
\text { lymphoma presenting with } \\
\text { intraoral symptoms }\end{array}$ & 1 & 4 & Branca & linfoma de burkitt & $\begin{array}{l}\text { lesões osteolíticas na } \\
\text { mandibula, mobilidade e dor } \\
\text { dentária }\end{array}$ \\
\hline 19 & Takinami & 1994 & Japão & $\begin{array}{l}\text { Radiation-induced hypoplasia } \\
\text { of the teeth and mandible }\end{array}$ & 1 & 4 & $\begin{array}{c}\text { Não } \\
\text { mencionado }\end{array}$ & $\begin{array}{l}\text { Hogroma cístico - } \\
\text { linfático }\end{array}$ & $\begin{array}{l}\text { hipoplasia dentária, } \\
\text { microdontia, exfoliação } \\
\text { precoce }\end{array}$ \\
\hline 20 & Katalin & 1995 & Hungria & $\begin{array}{l}\text { Dental complications of } \\
\text { radiotherapy of tumors of the } \\
\text { nasal cavity in childhood }\end{array}$ & 1 & 4 & $\begin{array}{l}\text { Não } \\
\text { mencionado }\end{array}$ & $\begin{array}{c}\text { Tumor } \\
\text { endodérmico seio } \\
\text { nasal }\end{array}$ & $\begin{array}{l}\text { dentes com amelogêneses e } \\
\text { dentinogêneses }\end{array}$ \\
\hline
\end{tabular}


Research, Society and Development, v. 10, n. 8, e35510817072, 2021

(CC BY 4.0) | ISSN 2525-3409 | DOI: http://dx.doi.org/10.33448/rsd-v10i8.17072

\begin{tabular}{|c|c|c|c|c|c|c|c|c|c|}
\hline 21 & Carl & 1980 & $\begin{array}{l}\text { Estados } \\
\text { Unidos }\end{array}$ & $\begin{array}{l}\text { Effects of radiation on the } \\
\text { developing dentition and } \\
\text { supporting bone }\end{array}$ & 2 & 4 e 9 anos & $\begin{array}{c}\text { Não } \\
\text { mencionado }\end{array}$ & rabdomiossarcoma & $\begin{array}{l}\text { cárie, falta de formaçao } \\
\text { radicular, } \\
\text { subdesenvolvimento } \\
\text { mandibula }\end{array}$ \\
\hline 22 & Kaste & 1994 & $\begin{array}{l}\text { Estados } \\
\text { Unidos }\end{array}$ & $\begin{array}{l}\text { Micrognathia after radiation } \\
\text { therapy for childhood facial } \\
\text { tumors. Report of two cases } \\
\text { with long-term follow-up }\end{array}$ & 2 & 4 e 5 anos & $\begin{array}{c}\text { Não } \\
\text { mencionado }\end{array}$ & rabdomiossarcoma & $\begin{array}{l}\text { hipoplasia, anodontia, } \\
\text { micrognatia }\end{array}$ \\
\hline 23 & Berkowitz & 1989 & $\begin{array}{l}\text { Estados } \\
\text { Unidos }\end{array}$ & $\begin{array}{l}\text { Developmental orofacial } \\
\text { deficits associated with } \\
\text { multimodal cancer therapy: } \\
\text { case report }\end{array}$ & 1 & $\begin{array}{l}2 \text { anos e } 5 \\
\text { meses }\end{array}$ & $\begin{array}{c}\text { Não } \\
\text { mencionado }\end{array}$ & rabdomiossarcoma & $\begin{array}{c}\text { agenesia dentária, mal } \\
\text { formação radicular, dentes } \\
\text { ectopico, coroa malformada }\end{array}$ \\
\hline 24 & Laudenbach & 1979 & França & $\begin{array}{l}\text { Disturbances in development of } \\
\text { the maxilla following } \\
\text { interstitial radium therapy for a } \\
\text { facial rhabdomyosarcoma at } \\
\text { the age of } 3 \text { years (author's } \\
\text { transl) }\end{array}$ & 1 & 3 & $\begin{array}{l}\text { Não } \\
\text { mencionado }\end{array}$ & rabdomiossarcoma & $\begin{array}{l}\text { mal formação radicular, } \\
\text { dente }\end{array}$ \\
\hline 25 & Weuman & 1968 & Inglaterra & $\begin{array}{l}\text { The effect of irradiation on } \\
\text { developing teeth }\end{array}$ & 1 & 3 & $\begin{array}{c}\text { Não } \\
\text { mencionado }\end{array}$ & nevus congenital & $\begin{array}{l}\text { dentes hipoplásicos, mal } \\
\text { formaçao radicular, }\end{array}$ \\
\hline 26 & Doline & 1980 & $\begin{array}{l}\text { Estados } \\
\text { Unidos }\end{array}$ & $\begin{array}{l}\text { The effect of radiotherapy in } \\
\text { the treatment of retinoblastoma } \\
\text { upon the developing dentition }\end{array}$ & 2 & 6 e 7 anos & $\begin{array}{c}\text { Não } \\
\text { mencionado }\end{array}$ & rabdomiossarcoma & $\begin{array}{l}\text { retrognatismo, carie, } \\
\text { desenvolvimento e coroa dos } \\
\text { molares anormais, mal } \\
\text { formação radicular, }\end{array}$ \\
\hline
\end{tabular}

Fonte: Autores. 
Research, Society and Development, v. 10, n. 8, e35510817072, 2021

(CC BY 4.0) | ISSN 2525-3409 | DOI: http://dx.doi.org/10.33448/rsd-v10i8.17072

Fazendo o panorama do câncer infantil, os mais frequentes são as leucemias, tumores no sistema nervoso central, no sistema linfático, nos rins, nos ossos e na retina. O diagnóstico e consequentemente o tratamento precoce aumenta a sua probabilidade em até 70\% (de Oliveira, DRM, Ramos, \& Curado, 2020). Nos estudos analisados nesta revisão, além das leucemias e tumores do sistema nervoso, observamos que as crianças também apresentaram tumores no tecido muscular estriado esquelético, mencionado em 8 artigos (Aielli et al., 2014; Berkowitz et al., 1989; Carl \& Wood, 1980; Doline, Needleman, Petersen, \& Cassady, 1980; Hwang \& Yoon, 2011; Jalali, Unkel, \& Reed, 2012; Kaste \& Hopkins, 1994; Laudenbach, Boudiere, \& Heubes, 1979).

Em todos os artigos, os autores citaram que o tratamento oncológico pode ocasionar possíveis complicações e efeitos colaterais nos pacientes, e que na criança pode ser na forma aguda ou crônica, uma vez que ela está na fase de crescimento e desenvolvimento (Juarez-Lopez, Solano-Silva, Fragoso-Rios, \& Murrieta-Pruneda, 2018; Ponce-Torres, Ruiz-Rodriguez Mdel, Alejo-Gonzalez, Hernandez-Sierra, \& Pozos-Guillen Ade, 2010). Outro ponto que houve uma concordância entre os pesquisadores foi sobre a ocorrência de complicações bucais em crianças ser de três vezes maior que em adultos, devido à alta atividade celular mitótica (Rimulo, Ferreira, Abreu, Aguirre-Neto, \& Paiva, 2011; Svoboda, Aaron, \& Albano, 1991).

Os efeitos das terapias antineoplásicas podem ser reversíveis (quimioterapia) ou progressivos e irreversíveis (radioterapia) e dependem de fatores do próprio paciente, tempo de tratamento, volume do tratamento, dose de distribuição e o uso concomitante com outras terapias (Avsar, Elli, Darka, \& Pinarli, 2007; Gandhi, Datta, Ahuja, Saxena, \& A, 2017). A saúde bucal e a saúde geral têm uma relação sinérgica e dinâmica em que a manutenção de uma boa saúde bucal é crítica para o sustento da saúde geral e vice-versa (Kapoor, Goswami, Sharma, Mehta, \& Dhillon, 2019). Analisando os artigos incluídos, as lesões bucais agudas mais prevalentes em crianças durante o tratamento foi a mucosite, ulcerações, sangramento espontâneo e candidíase. Lesões essas dolorosas, porém já estabelecidas na literatura cientifica (Juarez-Lopez et al., 2018; Kowlessar et al., 2019; Lucena, Damascena, Ribeiro, Lima-Filho, \& Valenca, 2019), para o manejo da mucosite, o cirurgião dentista pode ajudar no tratamento, que pode ser dividida em: suporte nutricional, controle da dor, descontaminação oral, paliação da boca seca, gerenciamento de sangramento oral e intervenções terapêuticas (Lalla, Sonis, \& Peterson, 2008). Outra alteração também observada nos artigos foi a presença da xerostomia, perda do paladar e hipossalivação (Jalali et al., 2012; Lucena et al., 2019, Velten, Zandonade, \& Monteiro de Barros Miotto, 2017). Todos esses fatores agem em conjunto para recorrência da mucosite, alteração no paladar, dificuldade na ingestão, mastigação e degustação dos alimentos, resultando em perda de peso (Cohen, Wakefield, \& Laing, 2016). Quando correlacionamos o tratamento oncológico com as manifestações bucais nas crianças, observamos a ocorrência de cárie e a gengivite, uma vez que há mudança na alimentação e diminuição na frequência da higiene bucal.

Portanto, a importância do acompanhamento com odontopediatria nesse grupo de crianças em tratamento é essencial para instruir o paciente quanto aos cuidados de higiene bucal durante todo o tratamento oncológico e quanto à sua manutenção posterior. Até porque o uso dos dentifrícios mais comuns é frequentemente suspenso, pois se torna intolerável para pacientes com mucosa oral inflamada, muitas vezes sofrendo também de disgeusia e xerostomia. O desconforto se deve principalmente aos componentes irritantes da maioria dos cremes dentais comerciais, como abrasivos, surfactantes, aromas, agentes antibacterianos. Cremes dentais leves sem mentol - que é um álcool monoterpênico cíclico com conhecidas características refrescantes e irritantes - foram introduzidos no mercado porque podem ser usados durante o tratamento e sempre, escolher aqueles que apresentam flúor acima de 1000 ppm (K. K. Cheng, Chang, \& Yuen, 2004; Lalla et al., 2008).

As evidências existentes nos estudos incluídos indicam que os medicamentos quimioterápicos no tratamento do câncer infantil estão associados a distúrbios do desenvolvimento dentário (agenesia dentária, descoloração dentária, retardo no desenvolvimento dentário, hipoplasia do esmalte, microdontia, apicificação precoce)(Carl \& Wood, 1980; C. F. Cheng, Huang, 
Tsai, Ko, \& Liao, 2000; Farsi, 2016; Fleming, 1991; Hwang \& Yoon, 2011; Kaste \& Hopkins, 1994; Katalin, Mihaly, \& Arpad, 1995; Peretz, Sarnat, \& Kharouba, 2014; Venkataraghavan, Patil, Guvva, Karthik, \& Bhandi, 2013; Zarina \& NikHussein, 2005). A dentição humana pode apresentar variações de tamanho, formas, estrutura, número e cor, determinadas geneticamente, mas grandemente influenciadas por fatores extrínsecos. Desta maneira, é evidente a ocorrência de diferentes desvios de desenvolvimento na dentição decídua e/ou permanente, não sendo rara sua frequência encontrada no dia a dia clínico, determinando problemas funcionais, como na mastigação e fonação, e estéticos. Após analise dos estudos incluídos neste trabalho, foi possível observar que as crianças submetidas ao tratamento de quimioterapia ou radioterapia podem apresentar manifestações bucais clínicas durante o tratamento e alterações de forma crônicas. Essa pesquisa inicial fez um panorama sobre as repercussões bucais que podem acometer em crianças submetidas ao tratamento contra os diversos tipos de câncer infantil. Como limitação, analisamos apenas artigos em língua inglesa, portanto, utilizamos apenas um banco de dados no qual encontraríamos texto em inglês. Mesmo pensando que tivemos uma escassez de materiais publicados encontrados, as evidências apontadas podem ser úteis para discussões sobre a temática, bem como para o desenvolvimento de futuras pesquisas de revisão sistemática incluindo outros banco de dados de pesquisa, além de literatura cinza, no qual poderão vir a acrescentar ou refutar os achados deste estudo. Assim sendo, esse artigo contribui de forma a ampliar o acesso à informação, reforçando o conhecimento dos profissionais e trazendo uma nova perspectiva sobre a importância do tema. Por esse estudo inicial, fica evidente que o odontopediatra possui uma atuação ampla e fundamental para o acompanhamento clinico de manifestações bucais em crianças submetidas no tratamento dos diversos tipos de câncer.

\section{Conclusão}

O recomendável é que antes mesmo de iniciar o tratamento oncológico, a criança passe por uma avaliação odontológica como maneira preventiva para tratar possíveis infecções já instaladas, fazer a devida adequação do meio bucal e receber orientações sobre a higiene oral, alimentação, cuidados e orientações sobre as possíveis alteração que a criança possa apresentar durante e após o tratamento.

\section{Referências}

Aielli, F., Giusti, R., Rughetti, A., dell'Orso, L., Ficorella, C., \& Porzio, G. (2014). Rapid resolution of refractory chemotherapy-induced oral mucositis with platelet gel-released supernatant in a pediatric cancer patient: a case report. J Pain Symptom Manage, 48(5), e2-4. 10.1016/j.jpainsymman.2014.06.015

Avsar, A., Elli, M., Darka, O., \& Pinarli, G. (2007). Long-term effects of chemotherapy on caries formation, dental development, and salivary factors in childhood cancer survivors. Oral Surg Oral Med Oral Pathol Oral Radiol Endod, 104(6), 781-789. 10.1016/j.tripleo.2007.02.029

Berkowitz, R. J., Neuman, P., Spalding, P., Novak, L., Strandjord, S., \& Coccia, P. F. (1989). Developmental orofacial deficits associated with multimodal cancer therapy: case report. Pediatr Dent, 11(3), 227-231. Retrieved from https://www.ncbi.nlm.nih.gov/pubmed/2638010

Carl, W., \& Wood, R. (1980). Effects of radiation on the developing dentition and supporting bone. J Am Dent Assoc, 101(4), 646-648. 10.14219/jada.archive.1980.0368

Carvalho, C. G., Medeiros-Filho, J. B., \& Ferreira, M. C. (2018). Guide for health professionals addressing oral care for individuals in oncological treatment based on scientific evidence. Support Care Cancer, 26(8), 2651-2661. 10.1007/s00520-018-4111-7

Cheng, C. F., Huang, W. H., Tsai, T. P., Ko, E. W., \& Liao, Y. F. (2000). Effects of cancer therapy on dental and maxillofacial development in children: report of case. ASDC J Dent Child, 67(3), 218-222, 161. Retrieved from https://www.ncbi.nlm.nih.gov/pubmed/10902084

Cheng, K. K., Chang, A. M., \& Yuen, M. P. (2004). Prevention of oral mucositis in paediatric patients treated with chemotherapy; a randomised crossover trial comparing two protocols of oral care. Eur J Cancer, 40(8), 1208-1216. 10.1016/j.ejca.2003.10.023

Chi, D. L. (2017). The Science and Art of Evidence-Based Pediatric Dentistry. Dent Clin North Am, 61(3), xi-xii. 10.1016/j.cden.2017.04.001

Cohen, J., Wakefield, C. E., \& Laing, D. G. (2016). Smell and Taste Disorders Resulting from Cancer and Chemotherapy. Curr Pharm Des, 22(15), 22532263. 10.2174/1381612822666160216150812

de Oliveira, M. M., DRM, E. S., Ramos, F. R., \& Curado, M. P. (2020). Children and adolescents cancer incidence, mortality and survival a population-based study in Midwest of Brazil. Cancer Epidemiol, 68, 101795. 10.1016/j.canep.2020.101795 
Doline, S., Needleman, H. L., Petersen, R. A., \& Cassady, J. R. (1980). The effect of radiotherapy in the treatment of retinoblastoma upon the developing dentition. J Pediatr Ophthalmol Strabismus, 17(2), 109-113. Retrieved from https://www.ncbi.nlm.nih.gov/pubmed/7391897

Effinger, K. E., Migliorati, C. A., Hudson, M. M., McMullen, K. P., Kaste, S. C., Ruble, K., \& Castellino, S. M. (2014). Oral and dental late effects in survivors of childhood cancer: a Children's Oncology Group report. Support Care Cancer, 22(7), 2009-2019. 10.1007/s00520-014-2260-x

Estrela, C. Metodologia cientifica: ciênica, ensino, pesquisa. Porto Alegre: Artes Médicas. 3ed. 2018.

Farsi, D. J. (2016). Children Undergoing Chemotherapy: Is It Too Late for Dental Rehabilitation? J Clin Pediatr Dent, 40(6), 503-505. 10.17796/1053-462840.6 .503

Fleming, P. (1991). Dental management of the pediatric oncology patient. Curr Opin Dent, 1(5), 577-582. Retrieved from https://www.ncbi.nlm.nih.gov/pubmed/1839663

Gandhi, K., Datta, G., Ahuja, S., Saxena, T., \& A, G. D. (2017). Prevalence of Oral Complications occurring in a Population of Pediatric Cancer Patients receiving Chemotherapy. Int J Clin Pediatr Dent, 10(2), 166-171. 10.5005/iD-iournals-10005-1428

Hwang, S. Y., \& Yoon, R. K. (2011). Developmental dental defects linked with chemoradiotherapy: a case report. J Clin Pediatr Dent, 35(3), 309-313. 10.17796/jcpd.35.3.t127p755m568x023

Jalali, G., Unkel, J. H., \& Reed, J. A. (2012). Management of dental complications in a child with rhabdomyosarcoma. Pediatr Dent, 34(7), 506-509. Retrieved from https://www.ncbi.nlm.nih.gov/pubmed/23265172

Juarez-Lopez, M. L. A., Solano-Silva, M. N., Fragoso-Rios, R., \& Murrieta-Pruneda, F. (2018). Oral diseases in children with acute lymphoblastic leukemia with chemotherapy treatment. Rev Med Inst Mex Seguro Soc, 56(2), 132-135. Retrieved from https://www.ncbi.nlm.nih.gov/pubmed/29901909

Kapoor, G., Goswami, M., Sharma, S., Mehta, A., \& Dhillon, J. K. (2019). Assessment of oral health status of children with Leukemia: A cross-sectional study. Spec Care Dentist, 39(6), 564-571. 10.1111/scd.12419

Kaste, S. C., \& Hopkins, K. P. (1994). Micrognathia after radiation therapy for childhood facial tumors. Report of two cases with long-term follow-up. Oral Surg Oral Med Oral Pathol, 77(1), 95-99. 10.1016/s0030-4220(06)80115-5

Katalin, G., Mihaly, O., \& Arpad, C. (1995). [Dental complications of radiotherapy of tumors of the nasal cavity in childhood]. Fogorv Sz, 88(12), 387-391. Retrieved from https://www.ncbi.nlm.nih.gov/pubmed/8713940

Kowlessar, A., Naidu, R., Ramroop, V., Nurse, J., Dookie, K., Bodkyn, C., \& Lalchandani, S. (2019). Oral health among children attending an oncology clinic in Trinidad. Clin Exp Dent Res, 5(6), 665-669. 10.1002/cre2.232

Lalla, R. V., Sonis, S. T., \& Peterson, D. E. (2008). Management of oral mucositis in patients who have cancer. Dent Clin North Am, 52(1), 61-77, viii. 10.1016/j.cden.2007.10.002

Laudenbach, P., Boudiere, J. P., \& Heubes, J. (1979). [Disturbances in development of the maxilla following interstitial radium therapy for a facial rhabdomyosarcoma at the age of 3 years (author's transl)]. Rev Stomatol Chir Maxillofac, 80(3), 174-177. Retrieved from https://www.ncbi.nlm.nih.gov/pubmed/288161

Lucena, N. N. N., Damascena, L. C. L., Ribeiro, I. L. A., Lima-Filho, L. M. A., \& Valenca, A. M. G. (2019). The Contribution of Motor Changes to Oral Mucositis in Pediatric Cancer Patients: A Cross-Sectional Study. Int J Environ Res Public Health, 16(18). 10.3390/ijerph16183395

Ludke, M. \& Andre, M. E. D. A. (2013). Pesquisas em educação: uma abordagem qualitativa. São Paulo: E.P.U.

Peretz, B., Sarnat, H., \& Kharouba, J. (2014). Chemotherapy induced dental changes in a child with medulloblastoma: a case report. J Clin Pediatr Dent, 38(3), 251-254. 10.17796/jcpd.38.3.c707838527x3155q

Ponce-Torres, E., Ruiz-Rodriguez Mdel, S., Alejo-Gonzalez, F., Hernandez-Sierra, J. F., \& Pozos-Guillen Ade, J. (2010). Oral manifestations in pediatric patients receiving chemotherapy for acute lymphoblastic leukemia. J Clin Pediatr Dent, 34(3), 275-279. 10.17796/jcpd.34.3.y060151580h301t7

Rimulo, A. L., Ferreira, M. C., Abreu, M. H., Aguirre-Neto, J. C., \& Paiva, S. M. (2011). Chemotherapy-induced oral mucositis in a patient with acute lymphoblastic leukaemia. Eur Arch Paediatr Dent, 12(2), 124-127. 10.1007/BF03262792

Ritwik, P., \& Chrisentery-Singleton, T. E. (2020). Oral and dental considerations in pediatric cancers. Cancer Metastasis Rev, 39(1), 43-53. 10.1007/s10555020-09842-5

Svoboda, W. E., Aaron, G. R., \& Albano, E. A. (1991). North American Burkitt's lymphoma presenting with intraoral symptoms. Pediatr Dent, 13(1), 52-58. Retrieved from https://www.ncbi.nlm.nih.gov/pubmed/1945986

Velten, D. B., Zandonade, E., \& Monteiro de Barros Miotto, M. H. (2017). Prevalence of oral manifestations in children and adolescents with cancer submitted to chemotherapy. BMC Oral Health, 17(1), 49. 10.1186/s12903-016-0331-8

Venkataraghavan, K., Patil, S., Guvva, S., Karthik, S., \& Bhandi, S. (2013). Abnormal odontogenesis following management of childhood cancer (retinoblastoma): review and a new variant. J Contemp Dent Pract, 14(2), 360-364. 10.5005/jp-journals-10024-1328

Zarina, R. S., \& Nik-Hussein, N. N. (2005). Dental abnormalities of a long-term survivor of a childhood hematological malignancy: literature review and report of a case. J Clin Pediatr Dent, 29(2), 167-174. 10.17796/jcpd.29.2.hq7307703428nt3v

Zubowska, M., Wyka, K., Fendler, W., Mlynarski, W., \& Zalewska-Szewczyk, B. (2013). Interleukin 18 as a marker of chronic nephropathy in children after anticancer treatment. Dis Markers, 35(6), 811-818. 10.1155/2013/369784 